\title{
Glucose-Lowering and the Risk of Cardiovascular Events with Novel Antidiabetic Therapies: A Systematic Review and Additive-effects Network Meta-Analysis
}

Luiz Sergio Fernandes de Carvalho

UNICAMP: Universidade Estadual de Campinas

Ana Claudia Cavalcante Nogueira

Escola Superior de Ciências da Saúde: Escola Superior de Ciencias da Saude

Riobaldo Marcelo Ribeiro Cintra

UNICAMP: Universidade Estadual de Campinas

Isabella Bonilha

UNICAMP: Universidade Estadual de Campinas

Beatriz Luchiari

UNICAMP: Universidade Estadual de Campinas

Alexander Benchimol

Instituto Estadual de Diabetes e Endocrinologia Luiz Capriglione

Carlos Eduardo Barra Couri

Universidade de São paulo

Jairo Lins Borges

Universidade Federal de São Paulo

Joaquim Barreto ( $\boldsymbol{D}$ joaquimbarretoantunes@gmail.com )

UNICAMP https://orcid.org/0000-0003-1914-316X

Andrei Carvalho Sposito

UNICAMP: Universidade Estadual de Campinas

\section{Research Article}

Keywords: New antidiabetic drugs, MACE, HbA1c

Posted Date: October 26th, 2021

DOl: https://doi.org/10.21203/rs.3.rs-983766/v1

License: (9) This work is licensed under a Creative Commons Attribution 4.0 International License.

Read Full License 
Page $2 / 19$ 


\section{Abstract}

Background: Among individuals with type 2 diabetes mellitus (T2DM), RCTs designed to investigate the cardiovascular effects of achieving $\mathrm{HbA} 1 \mathrm{c} \leq 7.0 \%$ by using insulin and sulfonylureas were unable to prevent the incidence of major adverse cardiovascular events (MACE) defined as CV death, non-fatal myocardial infarction, and non-fatal stroke. Intense glucose-lowering with newer antidiabetic therapies (ADTs) including SGLT2i, GLP1-RA, pioglitazone and DPP4i show lower risk of hypoglycemia and could lead to additive effect in preventing MACE. In this context, this study was designed to assess the impact of the HbA1c levels achieved with newer ADTs on the risk of MACE.

Methods. We searched MEDLINE/PubMed, Cochrane and ClinicalTrials.gov. RCTs published up to January/2021 reporting the occurrence of MACE and all-cause mortality in individuals with T2DM, including a sample size $\geq 100$ individuals in each study arm and follow-up $\geq 24$ weeks, were selected. Data was extracted by four independent observers following PRISMA guidelines. We performed a systematic review and additive-effects network meta-analysis with random effects and a multivariate meta-regression to assess the impact of achieved $\mathrm{HbA} 1 \mathrm{c}$ on incident MACE.

Results. A total of 122 RCTs were included with 139 treatment arms, 256,990 individuals, and 689,346 individuals-years who were randomized to an active treatment vs. control group. Therapy with SGLT2i, GLP1-RA, or pioglitazone similarly reduced the risk of MACE compared to placebo (HR $0.88[95 \% \mathrm{Cl} 0.83$, 0.94, $p<0.001$ ], 0.89 [95\% Cl 0.85, 0.94, $p<0.0001$ ], and 0.86 [95\% $\mathrm{Cl} 0.76,0.98, p=0.025$ ], respectively). The achievement of HbA1c $\leq 7.0 \%$ in RCTs with SGLT2i, DPP4i, TZD, or GLP1-RA in the active arm was associated with an adjusted $\mathrm{HR}$ of $0.91(95 \% \mathrm{Cl} 0.80,0.97 ; \mathrm{p}=0.039)$ compared with $\mathrm{HbA} 1 \mathrm{c}>7.0 \%$. Allcause mortality was not influenced by $\mathrm{HbA} 1 \mathrm{c}$ thresholds.

Conclusions: Achieving lower glucose levels with newer ADTs is linearly associated with a reduced risk of MACEs, without affecting all-cause mortality. Targeting HbA1c between 6.5 and $7 \%$ with SGLT2i, GLP1A, pioglitazone or DPP4i brings cardiovascular benefits considering the available RCT evidence.

\section{Study registration: PROSPERO CRD42020200649}

\section{Introduction}

Among individuals with type 2 diabetes mellitus (T2DM), observational studies have shown an increased risk of both macrovascular and microvascular events with increasing blood glucose levels ${ }^{1,2}$. Consistently, randomized clinical trials (RCTs) involving subjects with new-onset ${ }^{3}$ or long-lasting ${ }^{4-6}$ T2DM showed that intensive glucose control, i.e. glycated hemoglobin $(\mathrm{HbA} 1 \mathrm{c}) \leq 7.0 \%(53 \mathrm{mmol} / \mathrm{mol})$, reduces the incidence of microvascular complications. Nevertheless, the RCTs that were designed to investigate the cardiovascular effects of achieving $\mathrm{HbA1c} \leq 7.0 \%$ were not able to identify a significant decrease in the incidence of macrovascular events ${ }^{4-6}$. Several factors may explain these negative results, including lower rates of major adverse cardiovascular events (MACE) in the standard arm than originally predicted and adverse effects of therapies such as insulin- and sulfonylurea-associated weight gain and, 
particularly, hypoglycemia ${ }^{7}$. A meta-analysis suggested that these drugs could marginally attenuate the incidence of MACE by about $6 \%$, but the statistical power was insufficient to verify this assumption ${ }^{8}$. Therefore, it remains unclear whether the lack of evidence of cardiovascular benefit with an $\mathrm{Hba} 1 \mathrm{c} \leq 7.0 \%$ is due to the inadequacy of the statistical power or due to the side effects of some older antidiabetic therapies $(\mathrm{ADTs})^{4-6}$.

ADTs such as sodium-glucose cotransporter inhibitors (SGLT2i), glucagon-like peptide-1 receptor agonists (GLP1A), and pioglitazone, reduce MACE ${ }^{9-11}$ via a broad spectrum of mechanisms, which may or may not be additive to their glucose-lowering effect. Moreover, these new therapies, as well as dipeptidyl peptidase-4 inhibitors (DPP4i) have a low risk of hypoglycemia as a common advantage compared to the old drugs. A large number of RCTs have been performed to prove safety with the new ADTs, and in these studies, a wide variability of $\mathrm{HbA} 1 \mathrm{c}$ change was observed; while in some active arms, the post-treatment $\mathrm{HbA} 1 \mathrm{c}$ was $>8.0 \%$; in others, the post-therapy $\mathrm{HbA} 1 \mathrm{c}$ was $<7.0 \%{ }^{12}$. This scenario enabled us to test the assumption that glucose lowering has an additive effect in preventing MACE when drugs not associated with hypoglycemia are used, that is, SGLT2i, DPP4i, GLP1A, or pioglitazone. Hence, this systematic review aimed to investigate the association between glucose lowering in T2DM and the incidence of MACE in two sets of data: (i) all available RCTs reporting MACE to achieve sufficient statistical power, and (ii) exclusively RCTs that used the new ADTs.

\section{Methods}

\section{Search Strategy and Study Eligibility}

This systematic review was carried out in accordance with the guidelines of the Preferred Reporting Items for Systematic Reviews and Meta-analyses for Network Meta-analysis (PRISMA-NMA) ${ }^{13}$. A detailed description of all the procedures is provided in the Supplemental Material. Briefly, the review was performed by searching keywords in the following databases: Medline (PubMed), ClinicalTrials.gov, Cochrane Central Register of Controlled Trials, Embase, European Union Clinical Trials Register, and World Health Organization (WHO) International Clinical Trials Registry Platform electronic database entries until January 2021. Briefly, studies were included in the meta-analysis if they met all of the following criteria: (1) randomized double-blinded controlled study design; (2) sample size $\geq 100$ individuals in each study arm; (3) follow-up $\geq 24$ weeks; and (4) report of major adverse cardiovascular events (MACE) and CV mortality in both control and intervention groups. We excluded phase 1 or 2 RCTs, studies in type 1 diabetes, and studies without adequate information on outcomes or without a control group. The metaanalysis was registered in the International Prospective Register of Systematic Reviews (CRD42020200649) and detailed search terms, data sources are available in Supplementary Material.

\section{Data extraction and quality assessment}

Data were extracted by four authors (A.C.C.N., R.M.R.C., I.B., and B.L.), and any inconsistencies were resolved after debate with the senior researchers (A.C.S. and L.S.F.C.). Extracted data included the name 
of first author, year of publication, sample size, duration of follow-up, patient characteristics (sex, age, race), duration of diabetes, active (or experimental) and comparative drug, history of cardiovascular events and heart failure, average systolic and diastolic blood pressure, weight, body mass index (BMI), glycated hemoglobin values (HbA1c), estimated glomerular filtration rate (eGFR), clinical outcomes, and adverse events.

\section{Data synthesis and statistical analyses}

The primary endpoint was defined as 3-point MACE according to the definition of the study, representing a combination of nonfatal myocardial infarction, nonfatal stroke, and cardiovascular death. Secondary endpoints were defined as (i) nonfatal myocardial infarction and (ii) all-cause death. We used an additive component network meta-analysis (CNMA) framework to perform an indirect comparison between the drugs. This model is based on the premise that the effect of a treatment combination is the sum of the effects of its components, which implies that the common components cancel each other out in comparisons. An additive CNMA model can be used to evaluate the influence of individual components and their combinations, in contrast to standard network meta-analyses (NMA), which considers that all existing (single or combined) treatments are different nodes in the network. The advantage of employing CNMA here is to identify potential sources of bias related to drug combinations.

Dichotomous variables are reported as percentages, while continuous variables are reported as mean \pm SD or median (interquartile range). To identify the potential effects of therapies on clinical outcomes, we calculated the hazard ratios (HRs) with random-effects CNMA. We assessed statistical heterogeneity between trials using the $\mathrm{I}^{2}$ statistic (with $95 \% \mathrm{Cls}$ ), which is derived from Cochran's Q [100 $\left.(\mathrm{Q}-\mathrm{df} \div \mathrm{Q})\right]$ and provides a measure of the proportion of overall variation attributable to between-trial heterogeneity. We investigated potential sources of heterogeneity between the RCTs through meta-regression analyses using the restricted maximum-likelihood estimator and the method by Knapp and Hartung ${ }^{14}$ for adjusting test statistics and confidence intervals.

We performed prespecified multivariate meta-regressions to address the anticipated heterogeneity among RCTs in meta-regressions and to address the imbalance between RCTs that achieved $\mathrm{HbA} 1 \mathrm{c}<7.0 \%$ vs. $\mathrm{HbA} 1 \mathrm{c}>7.0 \%$ at the study end in the active arm. The adjusting variables were defined after data collection and were chosen if imbalance was identified between achieved HbA1c subgroups, namely: (i) the time since the diagnosis of T2DM, (ii) type of ADT in the active arm, and (iii) the length of follow-up time. To test for publication bias, we created funnel charts and performed the Egger test.

Sensitivity analyses were carried out to evaluate the stability of meta-regression models after: (i) to address whether largest trials are critical to results presented as main analyses, we sequentially excluded trials with the largest exposures (sample size * follow-up time); (ii) exclusion of the ACCORD, VADT, and ADVANCE trials ${ }^{4-6}$, RCTs that did not compare drug classes, but specific HbA1c targets. To estimate the effect of the treatment, a two-tailed p-value less than 0.05 , was considered statistically significant. Posthoc statistical power estimation was carried out using the method described by Jackson et al ${ }^{15}$. The 
extracted data were analyzed using R v4.0.1 (2020, Auckland, New Zealand) and discomb, metaviz, and metafor packages.

\section{Results}

We identified 3878 citations using the search terms and platforms mentioned above. After excluding duplicates, 3308 articles remained. We further excluded 623 articles that were unsuitable according to the title and abstract. An additional 2572 studies were excluded after full-text evaluation, with no results reported, open-label studies, post-hoc analyses, comparisons of the same drug classes, $n<100$ per group and/or treatment duration $<24$ weeks (details on Supplement data and Figure 1). We ended this extraction with 228 trials to be included for qualitative synthesis and meta-analysis, but among them, only 122 RCTs reported MACE. The flow diagram of the selection process and study network is shown in Figure 1 and in the Supplementary material, Figure S1, respectively.

These 122 RCTs provided data of 256,990 patients within 139 active arms, mean $1.48 \pm 1.16$ years of follow-up and total 689,346 patient-years that were randomized to an active treatment vs. control (full description of study arms is available in Supplements). The baseline characteristics of the enrolled individuals within the trials are shown in Supplementary Table S1. All studies presented a low risk of bias as assessed by the Cochrane Collaboration tool for assessing risk of bias ${ }^{16}$ (see Supplementary material, Table S2) and were deemed high quality by the GRADE system ${ }^{17}$, except for two RCTs: the CONFIDENCE trial and Abdul-ghani (2017). As shown in Supplementary Table S3 and Figure S2, there was no significant publication bias in the funnel plots, and there was no significant small study bias in the Egger tests.

The mean age was $57.3 \pm 9.6$ years ( $45 \%$ female). Individuals had the diagnosis of diabetes for $7.5 \pm 5.7$ years, $80 \%$ were on metformin at baseline, $22 \%$ were on insulin, $50.4 \%$ were enrolled in trials with individuals with prior $\mathrm{MI}$ or stroke at baseline and $13 \%$ of the enrolled individuals had heart failure at baseline. The mean baseline body mass index (BMI) across trials was $31.2 \pm 5.4 \mathrm{~kg} / \mathrm{m}^{2}$, body weight was $86.5 \pm 18.7 \mathrm{~kg}$, systolic blood pressure (SBP) was $131.1 \pm 6 \mathrm{mmHg}$, diastolic blood pressure (DBP) was $78.1 \pm 5 \mathrm{mmHg}$, estimated glomerular filtration rate was $81.09 .3 \mathrm{ml} / \mathrm{min} / 1.73 \mathrm{~m}^{2}$ and $\mathrm{HbA} 1 \mathrm{c} 8.1 \pm 0.9 \%$.

\section{Primary outcome}

The primary outcome (MACE) occurred in 9,457 individuals assigned to active treatment (median across trials of 13.26/1,000 patient-years [interquartile range (IQR) 15.5]) and 9,721 individuals assigned to the control group (median of 16.02/1,000 patient-years [IQR 16.2]). In an additive model network metaanalysis with random effects, DPP4i alone $(p=0.82)$, insulin $(p=0.23)$, sulfonylurea alone $(p=0.25)$, and metformin alone $(p=0.20)$ showed a neutral effect on the risk of MACE compared to placebo with no heterogeneity $\left(Q=94.5 ; I^{2}=0 \%, p=0.9884\right)$. Contrarily, SGLT2i alone, GLP1A alone, and pioglitazone alone 
reduced the risk of MACE compared to placebo with an $\mathrm{HR}$ of $0.88[95 \% \mathrm{Cl} 0.83,0.94, \mathrm{p}<0.001,0.89[95 \%$ $\mathrm{Cl} 0.85,0.94, p<0.0001]$ and 0.86 [95\% $\mathrm{Cl} 0.76,0.98, p=0.025]$, respectively) (Supplemental Figure S3). The meta-analysis with and without additive effects yielded similar results ( $p$ for difference 0.71 ).

\section{Secondary outcomes}

In order to evaluate the specificity of the findings, as secondary outcomes, we evaluated the relationship between HbA1c change and levels and secondary outcomes: (i) the HR for all-cause deaths and (ii) the HR for myocardial infarction. In meta-regression analyses, no relationship was found between the two secondary outcomes and achieved $\mathrm{HbA} 1 \mathrm{c}$ at the study end (Supplementary Figures S5a and S5b), nor with the differential change in $\mathrm{HbA} 1 \mathrm{c}$ between the active and control arms. Again, both the meta-analysis with and without additive effects showed similar effect ( $p$ for difference 0.91 for all-cause death and 0.56 for myocardial infarction).

\section{Meta-regression analyses}

As predicted during the study design, we observed that the RCTs contrasted largely in terms of glucoselowering efficacy, with absolute reductions in $\mathrm{HbA} 1 \mathrm{c}$ varying between $-2.2 \%$ and 0 in the active arms compared to their respective control arms. We performed a prespecified bivariate meta-regression analysis with all 139 study arms, including DPP4i, pioglitazone, GLP1A, insulin, metformin, sulfonylurea, and SGLT2 $\mathrm{i}$ in the active arms. RCTs reporting post-treatment $\mathrm{HbA} 1 \mathrm{c} \leq 7.0 \%$ were not associated ( $p$ for difference $=0.39 ; l^{2}=3.3 \%$ ) with the risk of MACE compared to those with $\mathrm{HbA} 1 \mathrm{c}>7.0 \%$ (Table 1). As a continuous variable, each $1 \%$ decrease in $\mathrm{HbA} 1 \mathrm{c}$ was also not associated ( $\mathrm{p}$ for difference $=0.12 ;\left.\right|^{2}=2.1 \%$ ) with the incidence of MACE (Table 1, Figure $2 b$ ). The achieved (post hoc) statistical power $(1-\beta)$ for comparing post-treatment $\mathrm{HbA} 1 \mathrm{c} \leq 7.0 \%$ vs $>7.0 \%$ was $87 \%$, considering the random-effect model, a two-tailed test and summary effect size of 0.0117 .

A second analysis was performed including only RCTs with SGLT2i, DPP4i, pioglitazone, or GLP1A in the active arm (Table 1). In this subgroup of RCTs, we found associations between MACE incidence and both $\mathrm{HbA} 1 \mathrm{c}$ values achieved after therapy and the absolute change in $\mathrm{HbA} 1 \mathrm{c}$; post-treatment $\mathrm{HbA} 1 \mathrm{c} \leq 7.0 \%$ was associated with $9 \%(95 \% \mathrm{Cl} 3,19 \%, \mathrm{p}=0.039)$ decrease in the risk of MACE compared with those achieving $\mathrm{HbA} 1 \mathrm{c}>7.0 \%$ (Figure $2 \mathrm{a}$ ); for every $1 \%$ reduction in $\mathrm{HbA} 1 \mathrm{c}$, there was an $18 \%$ reduction $(95 \% \mathrm{Cl}$ $7 \%, 29 \%, p<0.001$ ) in the risk of MACE (Figure $2 b$ ).

The pattern of the association between $\mathrm{HbA} 1 \mathrm{c}$ and MACE was investigated to estimate the existence of a threshold for the loss of benefit. Through polynomial meta-regression, we identified linear regression as the best fit for the association between MACE and the level of $\mathrm{HbA} 1 \mathrm{c}$ or the magnitude of the change in 
$\mathrm{HbA1c}$ after therapy, which does not suggest the existence of $\mathrm{U}$ or $\mathrm{J}$ curves for this association up to $\mathrm{HbA} 1 \mathrm{c}$ levels between $6.5 \%$ and $7.0 \%$. HbA1c levels $\leq 6.5 \%$ were not found in the RCTs.

Among all included RCTs, among those that reported post-treatment values of $\mathrm{HbA} 1 \mathrm{c} \leq 7.0 \%$, we found more frequently studies that included patients with shorter T2DM duration $(p=0.03)$, with longer follow-up $(p=0.01)$ and with GLP1A as their active arm $(p=0.03)$. These three variables were independently associated with the risk of developing MACEs. To circumvent these limitations, we performed multivariate meta-regressions for the risk of MACE adjusting for these covariates and, as shown in Table 1, each 1\% decrease in $\mathrm{HbA} 1 \mathrm{c}$ was associated with an HR for MACE of $0.90(95 \% \mathrm{Cl} 0.75,0.98, \mathrm{p}=0.017,12=0 \%)$ among RCTs with SGLT2i, DPP4i, pioglitazone, or GLP1A in the active arm. Likewise, the association between $\mathrm{HbA} 1 \mathrm{c} \leq 7.0 \%$ after treatment was also associated with an HR for MACE of $0.82(95 \% \mathrm{Cl} 0.70$, $0.96, p=0.013,12=0 \%)$ in the adjusted analysis.

\section{Sensitivity analyses}

Sensitivity analyses were conducted using three approaches: (i) the exclusion of one study with the largest exposures (sample size * follow-up time) per drug in the active arm, (ii) the exclusion of two studies per drug group with the largest exposures, and (iii) the exclusion of ACCORD, ADVANCE, and VADT trials. The first two approaches address the impact of potential class-related mechanisms on the relationship between achieved $\mathrm{HbA} 1 \mathrm{c}$ and the risk of MACE, and the third approach addresses the potential influence of the use of the old therapies with a higher risk of hypoglycemia.

To exclude that largest trials are critical to results presented as main analyses, in the first sensitivity analyses we excluded the trials TECOS, REWIND, DECLARE, and Dormandy (2005). As shown in Supplementary Figure S4a, in an additive model network meta-analysis with random-effects, only SGLT2i alone and GLP1A alone and the associations SGLT2 $i$ + GLP1A and SGLT2 $i$ + DPP4 were associated with reduced risk of MACE compared to placebo with HRs of 0.85 (95\% Cl 0.79, 0.92, p<0.0001), 0.89 ( $95 \% \mathrm{Cl}$ $0.85,0.94, p<0.0001), 0.76(95 \% \mathrm{Cl} 0.69,0.83, p<0.0001)$ and $0.84(95 \% \mathrm{Cl} 0.76,0.93, p=0.001)$, respectively, and no heterogeneity $\left(\mathrm{I}^{2}=0 \%\right)$.

In the second sensitivity analysis, we excluded the TECOS, CAROLINA, REWIND, EXCEL, DECLARE, CANVAS, and Dormandy (2005), and the results were unchanged compared to the first sensitivity analysis, and no heterogeneity was found $\left(I^{2}=0 \%\right)$ (Supplementary Figure $\left.S 4 b\right)$. A meta-regression based on this second approach yielded similar results when compared with the pre-exclusion dataset. There were significant relationships between the risk of MACE and the achieved $\mathrm{HbA} 1 \mathrm{c}$ levels in the active arm or the differential change in $\mathrm{HbA} 1 \mathrm{c}$ in the active arm compared to the control (Table 2).

In the third sensitivity analysis, we found no significant changes in the relationship between the risk of MACE and the achieved HbA1c levels in the active arm or the differential change in $\mathrm{HbA1c}$ when we excluded the ACCORD, ADVANCE, and VADT trials (data not shown). 


\section{Discussion}

The present systematic review and meta-analysis evaluated 122 RCTs with over 689,000 individualsyears and showed that the absolute change in $\mathrm{HbA} 1 \mathrm{c}$ and the target $\leq 7.0 \%$ were associated with reduced risk of MACE in therapies based on SGLT2i, DPP4i, pioglitazone, or GLP1A, with no evidence of increasing all-cause mortality.

Our results are in line with previous meta-analyses 812,18 and showed that the absolute change in $\mathrm{HbA} 1 \mathrm{c}$ and an achieved $\mathrm{HbA} 1 \mathrm{c} \leq 7.0 \%$ in patients with $\mathrm{T} 2 \mathrm{DM}$ is associated with mild reductions in MACE risk. In disagreement with our findings, Wang et al ${ }^{18}$ pooled 15 studies with 88,266 type 2 diabetes individuals and reported that an $\mathrm{HbA} 1 \mathrm{c}<7.0 \%$ did not improve cardiovascular outcomes. It is worth mentioning that their meta-analysis yielded high heterogeneity and pooled various study designs, including trials with post-acute coronary syndromes such as DIGAMI- $1^{19}$. Our differential approach in this meta-analysis was to carry out a more comprehensive literature search and, therefore, with greater statistical power and evaluated the newer ADTs independently.

Notably, although our meta-analysis did not capture significant heterogeneity among RCTs regarding the risk of MACE within ADT classes, there was a marked imbalance in baseline characteristics of individuals enrolled in RCTs with $\mathrm{HbA} 1 \mathrm{c} \leq 7.0 \%$ compared to RCTs that achieved HbA1c $>7.0 \%$. RCTs achieving $\mathrm{HbA} 1 \mathrm{c}>7.0 \%$ were shorter in duration and more frequently enrolled individuals with long-term T2D (> 8-10 years of disease). Although this is expected, no prior meta-analysis adjusted the regressions for these important cofactors ${ }^{12,18}$. Hence, in this study, we performed a step forward using multivariate metaregression analyses adjusting for T2DM duration, follow-up duration, and the effect of treatment in the active arm, and confirmed that achieving $\mathrm{HbA} 1 \mathrm{c} \leq 7.0 \%$ with SGLT2i, GLP1A, DPP4i, or pioglitazone was associated with a decreased risk of MACE compared to $>7.0 \%$.

Some findings from this meta-analysis indicate a potential contribution of blood glucose lowering in the reduction of macrovascular events. With the new ADTs, RCTs with post-therapy HbA1c $\leq 7.0 \%$ were consistently associated with a $9 \%$ lower risk of MACE compared with RCTs that achieved $\mathrm{HbA} 1 \mathrm{c}>7.0 \%$, regardless of the therapies used. A linear trend was found between MACE and $\mathrm{HbA} 1 \mathrm{c}$ in the range of 6.5$8.0 \%$, with no evidence of $\mathrm{U}$ - or $\mathrm{J}$-shaped curves and in a magnitude of association that is similar to that reported between hyperglycemia and MACE in observational studies ${ }^{1,2}$. We plan to verify this association by a meta-regression exclusively based on DPP4i, whose RCTs demonstrated a low risk of hypoglycemia, no weight gain, and no direct cardiovascular benefit. However, the sample size with the combination of these RCTs did not provide sufficient statistical power for this analysis. We found a very similar effect of SGLT2i, GLP1RA, and pioglitazone in the decrease of MACE risk. As commented above, these effects result from a wide range of mechanisms, which are concomitant and difficult to dissociate from their glucose-lowering effects. Thus, although this meta-analysis indicates the existence of a direct effect of lowering blood glucose levels on the incidence of MACE, our data do not allow us to determine the exact size of this effect. 
Our study had limitations. First, our results were obtained by meta-regression analysis from RCTs, which is inferior to analyses at the patient level. Nevertheless, in sensitivity analyses, when we evaluated different scenarios, excluding trials with larger exposures, we noticed similar results. Second, as mentioned above, the new ADTs have demonstrated MACE risk-reduction mechanisms that are independent of glycemic control or hypoglycemia. Therefore, the available data do not allow us to accurately estimate the magnitude of the effect of lowering blood glucose levels in reducing MACE.

In summary, more intense reductions in $\mathrm{HbA} 1 \mathrm{c}$ and lower levels of $\mathrm{HbA} 1 \mathrm{c}$ achieved with newer $\mathrm{ADTs}$ are associated with a reduced risk of MACE. Targeting HbA1c between $6.5 \%$ and $7 \%$ with SGLT2i, GLP1A, pioglitazone, or DPP4i may be associated with cardiovascular risk reduction in light of the available RCT evidence.

\section{Abbreviations}

MACE

major acute cardiovascular events

T2DM

type 2 diabetes mellitus

$\mathrm{RCT}$, randomized clinical trials

$\mathrm{HbA1c}$

glycated hemoglobin A1C

$\mathrm{CV}$, cardiovascular

$\mathrm{ADT}$, antidiabetic therapies

SGLT2i, sodium-glucose cotransporter inhibitors

GLP1A, glucagon-like peptide-1 receptor agonists

DPP4i, dipeptidyl peptidase-4 (DPP4) inhibitors

HR, hazard ratio

PRISMA, Preferred Reporting Items for Systematic Reviews and Meta-analyses for Network Meta-analysis BMI, body mass index

eGFR, estimated glomerular filtration rate

CNMA, additive component network meta-analysis

NMA, network meta-analysis

IQR, interquartile range.

\section{Declarations}

Ethics approval and consent to participate. Not applicable.

Consent for publication. Not applicable. 
Availability of data and materials. The data that support the findings of this study are available on request from the corresponding author, ACS.

Competing Interest. The authors have declared that no conflict of interest exists.

Funding: This work was supported by grants 437413/2018-7 and 301465/2017-7 from the Brazilian National Research Council (CNPq), and 2019/09068-3 from the São Paulo State Foundation for Research (FAPESP).

Author Contributions: Professor Carvalho had full access to all the data in the study and takes responsibility for the integrity of the data and the accuracy of the data analysis. Concept and design: Benchimol, Couri, Borges, Sposito and Carvalho. Acquisition, analysis, or interpretation of data: Bonilha, Luchiari, Cintra, Barreto, Nogueira, Benchimol, Couri, Borges, Sposito, and Carvalho. Drafting of the manuscript: Sposito, Carvalho. Critical revision of the manuscript for important intellectual content: All authors. Statistical analysis: Carvalho. Supervision: Sposito, Carvalho.

Acknowledgements. Not applicable.

\section{References}

1. Khaw KT, Wareham N, Bingham S, Luben R, Welch A, Day N. Association of hemoglobin A1c with cardiovascular disease and mortality in adults: the European prospective investigation into cancer in Norfolk. Ann Intern Med. 2004;141(6):413-20.

2. Selvin E, Marinopoulos S, Berkenblit G, et al. Meta-analysis: glycosylated hemoglobin and cardiovascular disease in diabetes mellitus. Ann Intern Med. 2004;141(6):421-31.

3. UK Prospective Diabetes Study (UKPDS) G. Intensive blood-glucose control with sulphonylureas or insulin compared with conventional treatment and risk of complications in patients with type 2 diabetes (UKPDS 33). Lancet. 1998;352(9131):837-53.

4. Action to Control Cardiovascular Risk in Diabetes. Study G, Gerstein HC, Miller ME, et al. Effects of intensive glucose lowering in type 2 diabetes. The New England journal of medicine 2008;358(24):2545-59. 
5. Duckworth W, Abraira C, Moritz T, et al. Glucose control and vascular complications in veterans with type 2 diabetes. The New England journal of medicine. 2009;360(2):129-39.

6. Advance Collaborative G, Patel A, MacMahon S, et al. Intensive blood glucose control and vascular outcomes in patients with type 2 diabetes. The New England journal of medicine. 2008;358(24):2560-72.

7. Skyler JS, Bergenstal R, Bonow RO, et al. Intensive glycemic control and the prevention of cardiovascular events: implications of the ACCORD, ADVANCE, and VA diabetes trials: a position statement of the American Diabetes Association and a scientific statement of the American College of Cardiology Foundation and the American Heart Association. Diabetes care. 2009;32(1):187-92.

8. Sposito AC, Berwanger O, de Carvalho LSF, Saraiva JFK. GLP-1RAs in type 2 diabetes: mechanisms that underlie cardiovascular effects and overview of cardiovascular outcome data. Cardiovasc Diabetol. 2018;17(1):157.

9. Marso SP, Daniels GH, Brown-Frandsen K, et al. Liraglutide and Cardiovascular Outcomes in Type 2 Diabetes. The New England journal of medicine. 2016;375(4):311-22.

10. Zinman B, Wanner C, Lachin JM, et al. Empagliflozin, Cardiovascular Outcomes, and Mortality in Type 2 Diabetes. The New England journal of medicine. 2015;373(22):2117-28.

11. Kernan WN, Viscoli CM, Furie KL, et al. Pioglitazone after Ischemic Stroke or Transient Ischemic Attack. N Engl J Med. 2016;374(14):1321-31.

12. Giugliano D, Maiorino MI, Bellastella G, Chiodini P, Esposito K. Glycemic Control, Preexisting Cardiovascular Disease, and Risk of Major Cardiovascular Events in Patients with Type 2 Diabetes Mellitus: Systematic Review With Meta-Analysis of Cardiovascular Outcome Trials and Intensive Glucose Control Trials. Journal of the American Heart Association. 2019;8(12):e012356.

13. Stroup DF, Berlin JA, Morton SC, et al. Meta-analysis of observational studies in epidemiology: a proposal for reporting. Meta-analysis Of Observational Studies in Epidemiology (MOOSE) group. Jama. 2000;283(15):2008-12.

14. Knapp G, Hartung J. Improved tests for a random effects meta-regression with a single covariate. Stat Med. 2003;22(17):2693-710.

15. Jackson D, Turner R. Power analysis for random-effects meta-analysis. Res Synth Methods. 2017;8(3):290-302.

16. Higgins JP, Altman DG, Gotzsche PC, et al. The Cochrane Collaboration's tool for assessing risk of bias in randomised trials. BMJ. 2011;343:d5928.

17. Guyatt GH, Oxman AD, Sultan S, et al. GRADE guidelines: 9. Rating up the quality of evidence. J Clin Epidemiol. 2011;64(12):1311-6.

18. Wang P, Huang R, Lu S, et al. HbA1c below $7 \%$ as the goal of glucose control fails to maximize the cardiovascular benefits: a meta-analysis. Cardiovasc Diabetol. 2015;14:124.

19. Malmberg K, Ryden L, Wedel H, et al. Intense metabolic control by means of insulin in patients with diabetes mellitus and acute myocardial infarction (DIGAMI 2): effects on mortality and morbidity. European heart journal. 2005;26(7):650-61. 


\section{Tables}

Table 1. Bivariate and multivariate meta-regression models with the hazard ratio for major adverse cardiovascular events (MACE) as dependent variable 
Bivariate analyses

\begin{tabular}{|c|c|c|}
\hline $\mathrm{HR}$ & $95 \% \mathrm{CI}$ & \\
\hline & Lower & Upper \\
\hline
\end{tabular}

Achieved HbA1c at study end in the active arm (HbA1c $\leq 7.0 \%$ vs HbA1c $>7.0 \%$ ) vs MACE

All trials (139 study arms;

$\mathrm{n}=256,990 ; 19,178$ events)

$\mathrm{I}^{2}$ (residual heterogeneity / unaccounted variability): 3.28\%, $\mathrm{QE}=81.3(\mathrm{p}=0.97)$; tau2 (estimated amount of residual heterogeneity): $0.010(\mathrm{SE}=0.022)$

SGLT2i, DPP4i, TZD or GLP1A in the active arm $0,9130 \quad 0,8098 \quad 0,9704 \quad 0.039$ (69 study arms*;

$\mathrm{n}=185,344 ; 15,528$ events)

$\mathrm{I}^{2}$ (residual heterogeneity / unaccounted variability): 10.39\%, QE = 51.14 ( $\left.\mathrm{p}=0.95\right)$; tau2 (estimated amount of residual heterogeneity): $0.010(\mathrm{SE}=0.022)$

Change in HbA1c in the active arm compared to control (each reduction of 1\%) vs MACE
All trials (139 study arms;
$0,8926 \quad 0,7414 \quad 1,0257 \quad 0.116$

$\mathrm{n}=256,990 ; 19,178$ events)

$\mathrm{I}^{2}$ (residual heterogeneity / unaccounted variability): 2.08\%, $\mathrm{QE}=91.3$ ( $\mathrm{p}=0.99$ ); tau2 (estimated amount of residual heterogeneity): $0.010(\mathrm{SE}=0.022)$

SGLT2i, DPP4i, TZD or GLP1A in the active arm $0,8241 \quad 0,7057 \quad 0,9318<0.001$ (69 study arms*;

$$
\mathrm{n}=185,344 ; 15,528 \text { events) }
$$

$\mathrm{I}^{2}$ (residual heterogeneity / unaccounted variability): 5.8\%, QE = $65.68(\mathrm{p}=0.99)$; tau2 (estimated amount of residual heterogeneity): $0.008(\mathrm{SE}=0.023)$

Multivariate analyses**

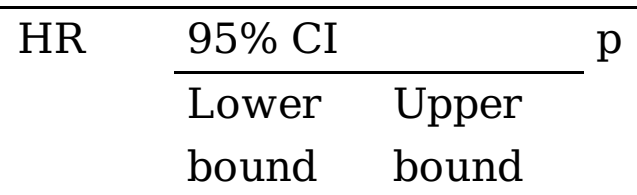

Achieved HbA1c at study end in the active arm (HbA1c $\leq 7.0 \%$ vs HbA1c $>7.0 \%$ ) vs MACE
All trials (118 study arms ${ }^{\S}$;
$0,86850,6263$
1,0964
0.186 $\mathrm{n}=231,071 ; 14,180$ events)

$\mathrm{I}^{2}$ (residual heterogeneity / unaccounted variability): 0\%, $\mathrm{QE}=71.4(\mathrm{p}=0.97)$; tau2 (estimated amount of residual heterogeneity): $0.014(\mathrm{SE}=0.012)$

SGLT2i, DPP4i, TZD or GLP1A in the active arm $0,8204 \quad 0,7019 \quad 0,95790.013$ (56 study arms*\$;

$\mathrm{n}=181,489 ; 14,876$ events)

$\mathrm{I}^{2}$ (residual heterogeneity / unaccounted variability): $0 \%, \mathrm{QE}=6.4(\mathrm{p}=0.21)$; tau2 
(estimated amount of residual heterogeneity): 0.010 (SE = 0.021)

Change in HbA1c in the active arm compared to control (each reduction of 1\%) vs MACE
All trials (116 study arms ${ }^{\S}$;
$0,87620,6992$
$1,0020 \quad 0.052$

$\mathrm{n}=228,852 ; 14,017$ events

$\mathrm{I}^{2}$ (residual heterogeneity / unaccounted variability): 0\%, $\mathrm{QE}=84.1(\mathrm{p}=0.99)$; tau2 (estimated amount of residual heterogeneity): 0.018 (SE = 0.027)

SGLT2i, DPP4i, TZD or GLP1A in the active arm 0,9047 0,7514 $0,9798 \quad 0.017$ (56 study arms*\$;

$\mathrm{n}=181,489 ; 14,876$ events)

$\mathrm{I}^{2}$ (residual heterogeneity / unaccounted variability): 0\%, $\mathrm{QE}=76.83(\mathrm{p}=0.98)$; tau2 (estimated amount of residual heterogeneity): $0.008(\mathrm{SE}=0.028)$

* Excluding RCTs with outlier HRs for MACE, defined as $\mathrm{HR} \geq 2.0$ or $\mathrm{HR} \leq 0.5$

** Adjusted for Time since the diagnosis of T2DM, follow-up time and type of ADT in the active arm. Adjusting variables were selected for their association with HR for MACE in bivariate analyses

$\S$ Some RCTs had missing data for covariates

Table 2. Sensitivity analyses ${ }^{¥}$ using meta-regression models for the hazard ratio of MACE as dependent variable 
Bivariate analyses

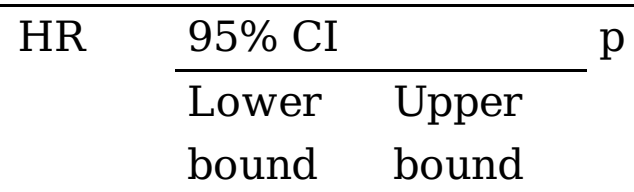

Achieved HbA1c at study end in the active arm (HbA1c $\leq 7.0 \%$ vs HbA1c $>7.0 \%$ ) vs MACE

All trials (132 study arms)

$0,9560 \quad 0,7703 \quad 1,1865 \quad 0.680$

$\mathrm{I}^{2}$ (residual heterogeneity / unaccounted variability): 3.87\%, $\mathrm{QE}=90.0(\mathrm{p}=0.99)$; tau2 (estimated amount of residual heterogeneity): $0.010(\mathrm{SE}=0.022)$

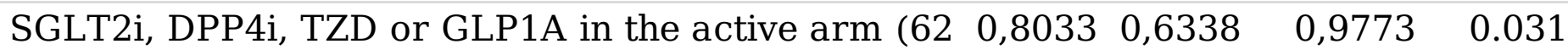
study arms*)

$\mathrm{I}^{2}$ (residual heterogeneity / unaccounted variability): 5.31\%, QE $=8.7(\mathrm{p}=0.37)$; tau2 (estimated amount of residual heterogeneity): $0.010(\mathrm{SE}=0.022)$

Change in HbA1c in the active arm compared to control (each reduction of 1\%) vs MACE
All trials (132 study arms)
$0,9036 \quad 0,7300 \quad 1,0526$
0.215

$\mathrm{I}^{2}$ (residual heterogeneity / unaccounted variability): 22.08\%, QE = $91.3(\mathrm{p}=0.99)$; tau2 (estimated amount of residual heterogeneity): $0.010(\mathrm{SE}=0.022)$

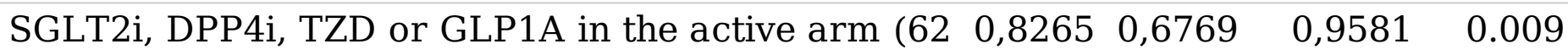
study arms*)

$\mathrm{I}^{2}$ (residual heterogeneity / unaccounted variability): 5.31\%, QE $=8.4(\mathrm{p}=0.35)$; tau2 (estimated amount of residual heterogeneity): $0.010(\mathrm{SE}=0.022)$

Multivariate analyses**

HR

\begin{tabular}{ll} 
95\% CI & \\
\hline Lower & Upper \\
bound & bound
\end{tabular}

Achieved HbA1c at study end in the active arm (HbA1c $\leq 7.0 \%$ vs HbA1c $>7.0 \%$ ) vs MACE

All trials (109 study arms ${ }^{\S}$ )

$0,8598 \quad 0,7082 \quad 1,0439$

0.073

$\mathrm{I}^{2}$ (residual heterogeneity / unaccounted variability): $0 \%, \mathrm{QE}=70.1$ ( $\left.\mathrm{p}=0.94\right)$; tau2 (estimated amount of residual heterogeneity): $0.014(\mathrm{SE}=0.012)$

SGLT2i, DPP4i, TZD or GLP1A in the active arm $(49 \quad 0,8328 \quad 0,7168 \quad 0,8755 \quad 0.004$ study arms*\$)

$\mathrm{I}^{2}$ (residual heterogeneity / unaccounted variability): 0\%, $\mathrm{QE}=5.6$ ( $\mathrm{p}=0.21$ ); tau2 (estimated amount of residual heterogeneity): $0.010(\mathrm{SE}=0.021)$

Change in HbA1c in the active arm compared to control (each reduction of 1\%) vs MACE
All trials (109 study arms ${ }^{\S}$ )
$0,8837 \quad 0,6765 \quad 1,0402$
0.107

$\mathrm{I}^{2}$ (residual heterogeneity / unaccounted variability): $0 \%, \mathrm{QE}=83.7$ ( $\mathrm{p}=0.97$ ); tau2 (estimated amount of residual heterogeneity): $0.018(\mathrm{SE}=0.027)$

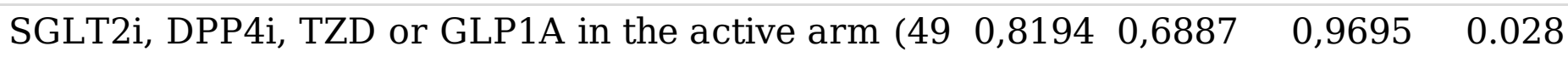


study arms*\$)

$\mathrm{I}^{2}$ (residual heterogeneity / unaccounted variability): $0 \%, \mathrm{QE}=6.1(\mathrm{p}=0.28)$; tau2 (estimated amount of residual heterogeneity): 0.008 (SE =0.028)

* Excluding RCTs with outlier HRs for MACE, defined as $\mathrm{HR} \geq 2.0$ or $\mathrm{HR} \leq 0.5$

** Adjusted for Time since the diagnosis of T2DM, follow-up time and type of ADT in the active arm. Adjusting variables were selected for their association with HR for MACE in bivariate analyses

$\S$ Some RCTs had missing data for covariates

$¥$ Data corresponds to sensitivity analysis 2 , which excluded the following RCTs: TECOS, CAROLINA, REWIND, EXCEL, DECLARE, CANVAS, and Dormandy (2005)

Figures 


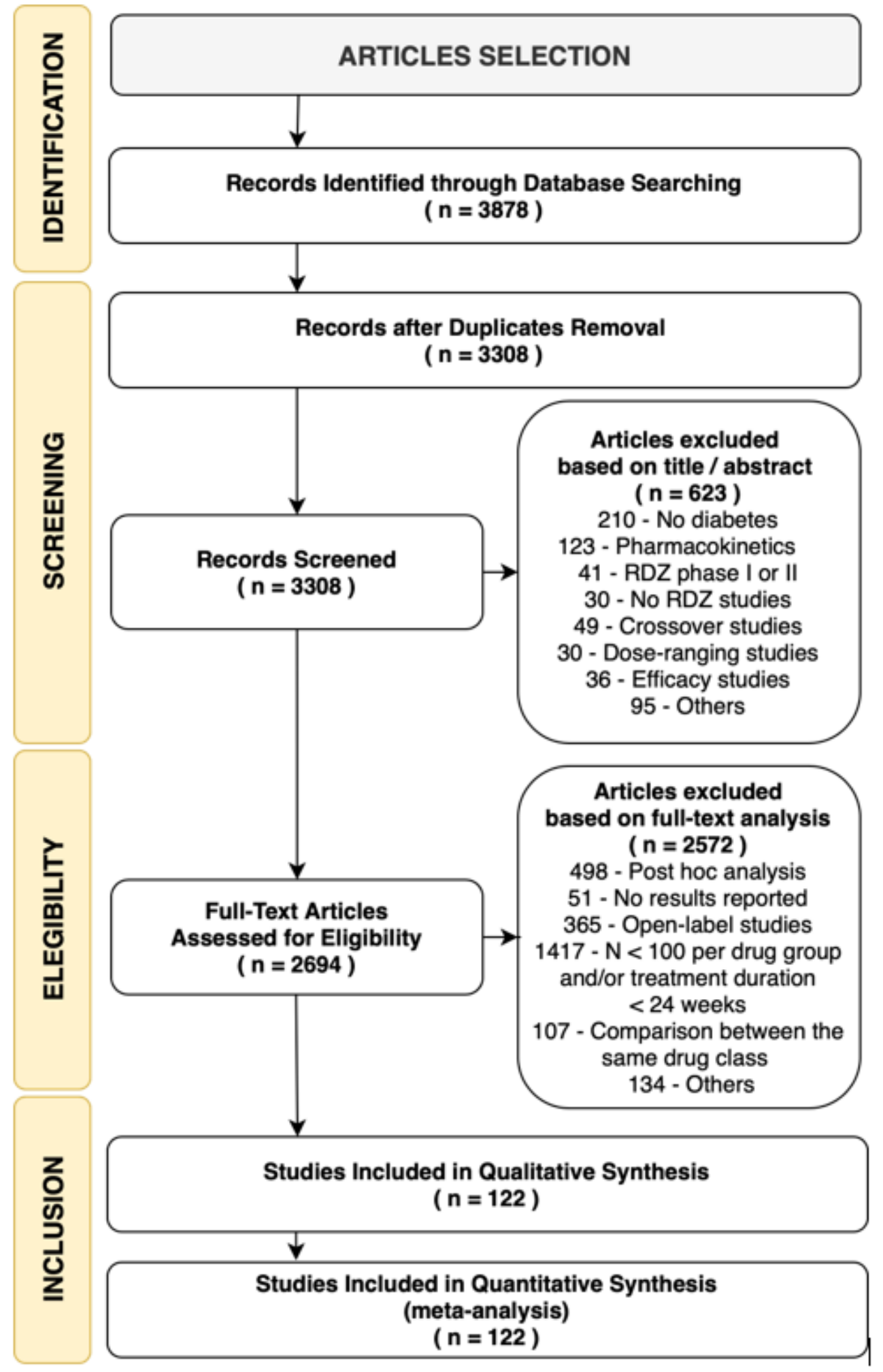

Figure 1

Flow diagram of the trials' selection process. RDZ: randomization. 

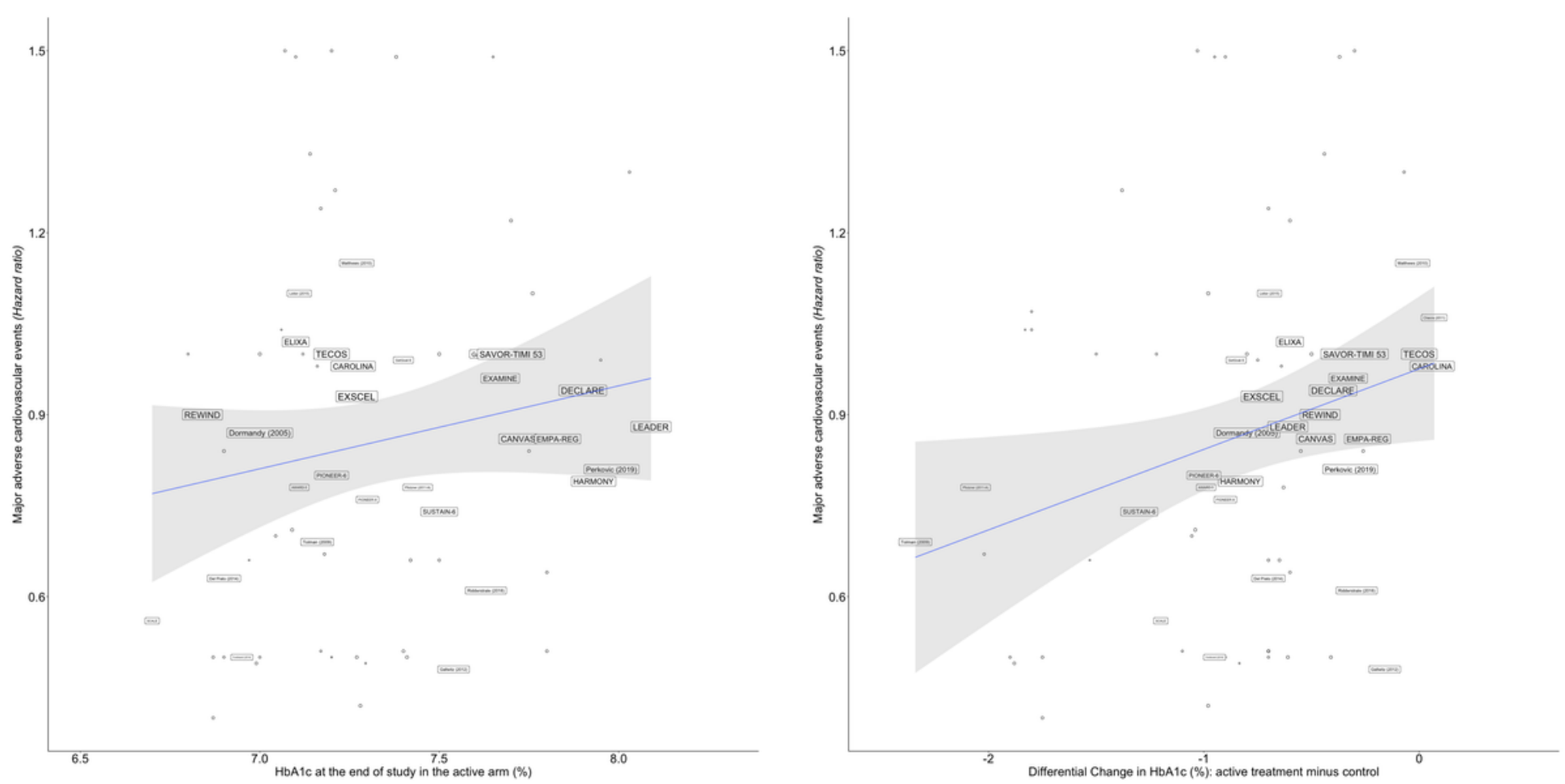

Figure 2

Meta-regression model for all drugs showing the relationship between the HR for MACE versus: (a) achieved $\mathrm{HbA} 1 \mathrm{c}$ levels at study end in the active arms of each trial, and (b) differential change in HbA1c levels between active and control arms. The size of the trials' name corresponds to their proportional weight in the regression.

\section{Supplementary Files}

This is a list of supplementary files associated with this preprint. Click to download.

- PRISMA2020checklist1.pdf

- Suppl20092021 copy.docx 